\title{
ON STABILITY AND HOPF BIFURCATIONS FOR CHEMOTAXIS SYSTEMS
}

\author{
E. N. DANCER ${ }^{\dagger}$
}

The purpose of this paper is to study the stability of peak solutions (boundary or interior peaks) for the chemotaxis system

$$
\begin{gathered}
\frac{\partial A}{\partial t}=\epsilon^{2} \Delta A-A+\frac{A^{p}}{\xi^{q}} \text { in } \Omega \\
\tau|\Omega| \frac{d \xi}{d t}=-|\Omega| \xi+\xi^{-s} \int_{\Omega} A^{r} d x \\
\frac{\partial A}{\partial n}=0 \text { on } \partial \Omega
\end{gathered}
$$

where $\epsilon>0, \tau>0, \Omega$ is a smooth bounded domain in $R^{N},|\Omega|$ denotes the measure of $\Omega, p>1, q>0, r>0, s \geq 0$ and we usually also assume $\gamma_{0} \equiv \frac{q r}{(p-1)(s+1)}>1$. This system is known as the shadow-system of the Gierer-Meinhardt system. The full Gierer-Meinhardt system is a quite regular perturbation of this system so it is nearly always very easy to deduce results for the full system (with a large parameter) from that of the shadow system. Now, as is well known, stationary solutions are determined by the equation

$$
\begin{gathered}
-\epsilon^{2} \Delta u=u^{p}-u \text { in } \Omega \\
u>0 \text { in } \Omega \\
\frac{\partial u}{\partial n}=0 \text { on } \partial \Omega
\end{gathered}
$$

by the substitution $A=\xi^{\frac{q}{p-1}} u(x)$ provided $\gamma_{0} \neq 1$. (If $\gamma_{0}=1$, there is either no solution or a curve of solutions, depending upon the other parameters). However, the parabolic system does not seem to reduce in such a simple way.

It is easy to see that the eigenvalue problem for the the linearization of (1) at a solution $A=\xi^{q(p-1)} u_{\epsilon}(x)$ where $\xi^{t}=|\Omega|^{-1} \int_{\Omega} u^{r}$ and $t=1+s-q r /(p-1)$ reduces to the eigenvalue problem

$$
\begin{gathered}
-\epsilon^{2} \Delta h+h-p u_{\epsilon}^{p-1} h+\hat{f}_{i, \epsilon \alpha}(h) u_{\epsilon}^{p}=\alpha h \text { on } \Omega \\
h=0 \text { on } \partial \Omega
\end{gathered}
$$

where $\hat{f}_{1, \epsilon, \alpha}(h)=\frac{q r}{s+1-\tau \alpha}\left(\int_{\Omega} u_{\epsilon}^{r}\right)^{-1} \int_{\Omega} u_{\epsilon}^{r-1} h$.

${ }^{\dagger}$ School of Mathematics and Statistics, University of Sydney, N.S.W. 2006, Australia (normd@maths.usyd.edu.au). Partially supported by the Australian Research Council. 
If $u$ is an interior peak solution of (2), Wei [10] proves that the much of the stability properties of the solution of (1), for small $\epsilon$, is determined by the number of eigenvalues $\alpha$ with negative real part of the problem

$$
\Delta \phi+\phi-p w^{p-1} \phi+\frac{q r}{s+1-\tau \alpha} \frac{\int_{R^{N}} w^{r-1} \phi}{\int_{R^{N}} w^{r}} w^{p}=\alpha \phi
$$

where $w$ is the positive radial decaying solution of $-\Delta u=u^{p}-u$ on $R^{N}$. More precisely, if all the non-zero eigenvalues of (4) have negative real part, one can prove that the corresponding solution of (1) is metastable, that is the only eigenvalue of the linearization of (1) with non-positive real part are small in $\epsilon$. (In the boundary peak case, the solutions may be stable). This implies that for small $\epsilon$ the solutions are stable for large times. Note that our eigenvalues have opposite sign to Wei's.

We prove more precise statements relating eigenvalues of (4) to these of (3), and further analyze (4). In particular, we show that one of the results of Wei [10] is essentially best possible (which is a little surprising since the proof in [10] is by quadratic forms). We also use the analysis of (4) to show that there can be a global generalized Hopf bifurcation of (1) to periodic solutions of (1) rather localized in space and time period neither small nor large. Ni, Takagi and Yanagida [9] proved a much more restrictive result of this type. They also obtain a number of related stability results on (1) by different arguments. We discuss this the relation of their work to ours in more detail later. Note that the results here, [9] and [10] are largely complementary.

In Section 1, we analyze (4). In Section 2, we relate (3) to (4) and look at Hopf bifurcations.

1. Analysis of the limiting equation. We analyze the problem (4) in the space $L^{2}\left(R^{N}\right)$ (with domain $H^{2}\left(R^{N}\right)$ ). We first show the system reduces. We write

$$
L^{2}\left(R^{N}\right)=L_{r}^{2}\left(R^{N}\right) \oplus W
$$

where $L_{r}^{2}\left(R^{N}\right)$ is the set of radical $L^{2}$ functions on $R^{N}$ and $W$ is its orthogonal complement (in $L^{2}\left(R^{N}\right)$ ). It is easy to see that $L_{\alpha}$, the left hand side of (4), maps $L_{r}^{2}\left(R^{N}\right) \cap H^{2}\left(R^{N}\right)$ into $L_{r}^{2}\left(R^{N}\right)$ and so this space is invariant. On the other hand, if $\phi \in W, \int_{R^{N}} w^{r-1} \phi=0$ and hence on this subspace $L_{\alpha} \phi=-\Delta \phi+\phi-p w^{p-1} \phi$ and it follows easily $L_{\alpha}$ maps $H^{2}\left(R^{N}\right) \cap W$ into $W$. (Remember that $-\Delta$ is self adjoint and maps $L_{r}^{2}\left(R^{N}\right) \cap H^{2}\left(R^{N}\right)$ into $L_{r}^{2}\left(R^{N}\right)$. Thus our equation (4) reduces to one on $L_{r}^{2}\left(R^{N}\right)$ and one on $W$. We sometimes write $L_{r}^{2}$ for $L_{r}^{2}\left(R^{N}\right)$. On $W$, our equation is

$$
-\Delta \phi+\phi-p w^{p-1} \phi=\alpha \phi
$$

which has zero as an eigenvalue of multiplicity $N$ and all the other eigenvalues (and other points of the spectrum) are real and positive. This is well known and follows for example from the argument on p970 of [2]. Note that the negative eigenvalue $\lambda_{1}$ of (5) (considered on $L^{2}\left(R^{N}\right)$ is simple with a radial eigenfunction. The zero eigenvalue of (5) on $W$ corresponds to the small eigenvalues which are discussed carefully in Wei. 
Thus our problem largely comes down to the study of the eigenvalue problem $L_{\alpha} \phi=\alpha \phi$ on $L_{r}^{2}$. Henceforth for this section, we work exclusively on $L_{r}^{2}$. Note that this is an ordinary differential equation rather than a partial differential equation. We always look at $\alpha$ with $\tau R e \alpha<s+1$. This includes the eigenvalues which determine stability.

Before discussing this in detail, we look at Fredholm properties. Consider the map $\phi \longrightarrow p w^{p-1} \phi+\gamma_{1}(\alpha) f_{1}(\phi) w^{p}$ (where $f_{1}(\phi)=\frac{\int_{R^{n}} w^{r-1} \phi}{\int_{R^{n}} w^{r}}$ and $\gamma_{1}(\alpha)=\frac{q r}{s+1-\tau \alpha}$ ). It is easily seen to be relatively compact as a map of $H^{2}\left(R^{N}\right)$ into $L^{2}\left(R^{N}\right)$. Hence $L_{\alpha}-\alpha I$ is Fredholm if and only if $-\Delta-(1-\alpha) I$ is Fredholm and this is true only if $\alpha<1$ or $\operatorname{Im} \alpha \neq 0$. Thus $L_{\alpha}-\alpha I$ is Fredholm of index zero if $\alpha<1$ or $\operatorname{Im} \alpha \neq 0$ and is not Fredholm otherwise.

Next note that, if $\alpha$ is an eigenvalue of $L_{\alpha} \phi=\alpha \phi$ on $L_{r}^{2}$ then its geometric multiplicity on $L_{r}^{2}$ is 1 unless $\alpha=\lambda_{1}$ or $\alpha$ is a positive eigenvalue of $L^{\prime}=-\Delta+(1-$ $\left.w^{p-1}\right) I$. To see this, note that if $w_{1}$ and $w_{2}$ are linearly independent eigenfunctions, a (possibly complex) linear combination $\widetilde{w}$ must satisfy $f_{1}(\widetilde{w})=0$. Thus $L^{\prime} \widetilde{w}=\alpha \widetilde{w}$ and $\widetilde{w} \in L_{r}^{2}$. Hence $\alpha$ is real (by self-adjointness of $L^{\prime}$ ) and $\alpha=\lambda_{1}$ or zero or is a positive eigenvalue of $L^{\prime}$. We will show $\alpha=0$ is impossible in a moment which proves our claim.

To complete the proof of the claim of the previous paragraph, we must study the eigenvalue zero on $L_{r}^{2}$. To do this, one writes our eigenvalue problem as

$$
\left(L^{\prime}-\alpha I\right) \phi=-\gamma_{1}(\alpha) f_{1}(\phi) w^{p} .
$$

Hence, if $\alpha$ is not an eigenvalue of $L^{\prime}$ on $L_{r}^{2}$,

$$
\phi=-\gamma_{1}(\alpha) f_{1}(\phi)\left(L^{\prime}-\alpha I\right)^{-1} w^{p}
$$

Hence if $\alpha$ is an eigenvalue of (4) on $L_{r}^{2}$ and $\phi$ is the corresponding eigenfunction, $f_{1}(\phi) \neq 0, \phi$ is a multiple of $\left(L^{\prime}-\alpha I\right)^{-1} w^{p}$ and taking $f_{1}$ of each side we see that

$$
\gamma_{1}(\alpha) f_{1}\left(\left(L^{\prime}-\alpha I\right)^{-1} w^{p}\right)=-1
$$

Conversely, if (8) is satisfied, it is easy to use (7) to check $\left(L^{\prime}-\alpha I\right)^{-1} w^{p}$ is an eigenfunction corresponding to $\alpha$. (8) is very useful. Our argument also shows the one dimensionality claimed earlier.

Now $L^{\prime}$ is invertible on $L_{r}^{2}$ and we easily see as in [10] then $L^{\prime} w=-(p-1) w^{p}$ that is, $\left(L^{\prime}\right)^{-1}\left(w^{p}\right)=-(p-1)^{-1} w$. Hence by (7), the definition of $f_{1}$ and a simple calculation, zero is an eigenvalue of (4) if and only if $\gamma_{0}=1$, that is $q r=(p-1)(s+1)$.

(8) also implies that (4) has no eigenvalues with $|\alpha|$ large and $\operatorname{Re} \alpha \leq K$. Firstly note that, such $\alpha$ are not in the spectrum of $L^{\prime}$ and in fact by standard resolvent estimates for self-adjoint operators (cp [6], eqn. V.3.16), $\left\|\left(L^{\prime}-\alpha I\right)^{-1}\right\| \leq \frac{1}{d\left(\alpha, \sigma\left(L^{\prime}\right)\right)} \longrightarrow$ 0 as $|\alpha| \longrightarrow \infty$ in this region. Since $\gamma(\alpha) \longrightarrow 0$ as $|\alpha| \longrightarrow \infty$ in this region, we easily see that left hand side of (8) tends to zero as $|\alpha| \longrightarrow \infty$ and hence (8) can not be satisfied. This proves our claim. It is easy to check that this estimates holds uniformly for $p, r, s, \tau$ in a compact set. For real $\alpha$ it is possible to use the positivity in the cone 
sense of $\left(L^{\prime}-\alpha I\right)^{-1}$ for $\alpha<\lambda_{1}$, to ensure $\alpha$ is not an eigenvalue of (4) if $\alpha$ is real and $\alpha<\lambda_{1}$. In fact, since $w^{p} \notin R\left(L^{\prime}-\lambda_{1} I\right)$ (where $\mathcal{R}$ denotes the range), one can also easily show that $\lambda_{1}$ is also never an eigenvalue of (4). As we will see later, (4) sometimes has complex eigenvalues.

Note that this, the analyticity of our operator in $\alpha$ for $\tau R e \alpha<1+s$, the Fredholm property for $R e \alpha<1$ and a theorem of Gokhberg and Krein [6, Theorem 3.6 ] imply that the eigenvalues of (4) in $\tau \operatorname{Re} \alpha<1+s$ and $\operatorname{Re} \alpha<1$ are isolated.

We need the notion of the multiplicity of an eigenvalue $\alpha$ of (1) with $\operatorname{Re} \alpha<1$ or $\operatorname{Im} \alpha \neq 0$ (where (4) is Fredholm of index zero). This is defined for example in Ize [5]. (Basically in the finite-dimensional case, the multiplicity of $\alpha_{0}$ is defined in terms of the multiplicity of $\alpha_{0}$ as a zero of the determinant, while in the infinite-dimensional case, we first do a Liapounov-Schmitt reduction to finite-dimensions. Note that while 0 always has geometric multiplicity in $L_{r}^{2}$ at most 1 , it sometimes has higher multiplicity, as we see below.

We will sometimes make use of the following remark. If we choose a continuous curve $y(t)$ in $(p, q, r, s)$ space so that there is no eigenvalue $\alpha$ with $\operatorname{Re} \alpha=0$ for any $y(t)$, then the sum of the multiplicities of the eigenvalues of the negative eigenvalues is independent of $t$. This uses our earlier bounds and properties of multiplicities under perturbation (cp. Dancer [3], Lemma 4 and the remark afterwards). Hence the stability can only change by an eigenvalue crossing the imaginary axis (as we vary $p, q, r, s)$. Moreover it is easy to check that if $\alpha$ is a complex eigenvalue, $\bar{\alpha}$ is also an eigenvalue and $\alpha$ and $\bar{\alpha}$ have the same multiplicity (since the coefficients in (4) are all real). Hence by our comments on multiplicity above, we see that if the sum of the multiplicities of the real negative eigenvalues is odd, this persists as we vary $p, q, r, s, \tau$ unless an eigenvalue becomes zero (since the sum of multiplicities of the eigenvalues with negative real part is always odd). This is a very useful instability result which we exploit below. Note that we only have a zero eigenvalue when $\gamma_{0}=1$.

To apply this, we need to examine the behaviour of small eigenvalues in $L_{r}^{2}$ as parameters vary. We first consider (4) with $\tau=0$. Note that while (1) is not well behaved for $\tau=0,(4)$ is well behaved. For $\tau=0,(4)$ is a standard eigenvalue problem and the multiplicity is then the standard algebraic multiplicity.

We now partially calculate this when $\gamma_{0}=1$ (which is the necessary and sufficient condition for zero to be an eigenvalue for $\tau=0$ ). As we saw earlier, the kernel of $L_{0}$ is then spanned by $w$. The algebraic multiplicity is 1 if $w$ is not in the range of $L_{0}$ (on $L_{r}^{2}$ ). Now, as Wei observed $L^{\prime}\left((p-1)^{-1} w+\frac{1}{2} R \frac{\partial w}{\partial R}\right)=-w$ (where $R=$ $|x|)$. Now if $L_{0} z=w$, then $L^{\prime} z+\gamma(0) f_{1}(z) w^{p}=w$. Since if $z$ is a solution so is $z+B w$ (since $L_{0} w=0$ ). Since $f_{1}(w) \neq 0$ by a simple computation, if there is a solution there is one with $f_{1}(z)=0$ and $L^{\prime}(z)=w$. Hence by our comment above $z=-\left((p-1)^{-1} w++\frac{1}{2} R \frac{\partial w}{\partial R}\right)$. Hence the algebraic multiplicity is at least 2 if and only if $f_{1}\left((p-1)^{-1} w+R \frac{\partial w}{\partial R}\right)=0$. By the definition of $f_{1}$ and a simple integration by parts, this becomes $(p-1)^{-1} \int w^{r}-\frac{N}{2 r} \int w^{r}=0$, that is $N(p-1)=2 r$. Hence we see that the algebraic multiplicity of zero is 1 if $\gamma_{0}=1$ and $N(p-1) \neq 2 r$ and is at least 2 if $\gamma_{0}=1$ and $N(p-1)=2 r$.

We do not know if an algebraic multiplicity of 3 or more is possible. (This reduces 
to discussing when the solution of $L^{\prime} z_{1}=(p-1)^{-1} w+\frac{1}{2} R \frac{\partial w}{\partial R}$ satisfies $f_{1}\left(z_{1}\right)=0$.) Note this is purely a condition on $r, p$ and $N$ and if it occurs it can be proved that it only occurs for isolated $r$ for fixed $N$ ). We will prove later indirectly that this does not occur for $r$ close to 2. (In other words the algebraic multiplicity is exactly 2 if $\gamma_{0}=1, N(p-1)=2 r$ and $r$ is close to 2.)

To prove the algebraic multiplicity of zero is indeed 2 for $\tau=0, r$ close to 2 , $\gamma_{0}=1, N(p-1)=2 r$, we need to make a bifurcation analysis of the small eigenvalues of $L_{0}$ for $\tau=0$ and $\mu$ near $\mu_{0}$. Here $\mu=\gamma(0)$ and $\mu_{0}=p-1$. To do this, we need to know the kernel of $L_{0}^{*}$. Now it is easy to prove that $L_{0}^{*}=L^{\prime}+\gamma_{0} f_{2}() w^{r-1}$ where $f_{2}(z)=\int_{R^{N}} w^{p} z / \int_{R^{N}} w^{r}$. (Note that $L_{0}^{*}$ is a similar type of operator to $L_{0}$ for different parameter values). We can argue much as before to deduce that the adjoint eigenfunction corresponding to the eigenvalue zero is $\hat{w}=\left(L^{\prime}\right)^{-1}\left(w^{r-1}\right)$. We examine the bifurcation equation using $\mu=\frac{q r}{s+1}$ as a parameter. We prove that the bifurcation equation for small eigenvalues of $L_{\alpha} \phi=\phi$ on $L_{r}^{2}$ for $\tau=0$ is of the form

$$
k \delta^{m}+k_{1} \mu^{\prime}+h o t=0
$$

where $k, k_{1} \neq 0$, the higher order terms hot are terms independent of $\mu^{\prime}$ and of smaller order than $\delta^{m}$ or are of $o\left(\mu^{\prime}\right)$ (and the corresponding properties of the derivatives) and $m$ is the algebraic multiplicity of zero. Here $\mu^{\prime}=\mu-\mu_{0}$ where zero is an eigenvalue for $\mu=\mu_{0}=p-1$.

Assuming this for a moment, we analyze the small eigenvalues. It follows easily from (9) that $\frac{\delta}{|\mu|^{\frac{1}{m}}}$ is close to a solution of $z^{m}+\frac{k_{1}}{k} s q n \mu=0$ if $\mu \neq 0$. By this and the implicit function theorem, we see that for small non-zero $\mu$ there are exactly $m$ small eigenvalues of $L_{0}$ and these are of the form $|\mu|^{\frac{1}{m}} \tau_{i}+o\left(|\mu|^{\frac{1}{m}}\right)$ where $\tau_{i}$ is a root of $z^{m}=-k_{1} / k$ if $\mu>0$ and is a root of $z^{m}=k_{1} / k$ if $\mu<0$ (for small $\mu$ ). Note that this means that, if $m>1$, asymptotically the arguments of the roots are equally spaced to first order. Note that since a complex conjugate of a solution is again a solution, the real solutions correspond to the real $\tau_{i}$.

Let us now consider cases. Assume first that $m=1$, that is the eigenvalue is simple for $\mu=\mu_{0}$. In this case, our analyses shows that there is a unique small eigenvalue for $\mu^{\prime}$ small, it is always real and it crosses the imaginary axis as $\mu^{\prime}$ crosses zero. Thus stability must fail on one side of zero. In particular if there are no purely imaginary eigenvalue at all for $\mu^{\prime}=0$, the number of real negative eigenvalues must change for even to odd as $\mu^{\prime}$ crosses zero. As we remarked earlier, this local instability for $\mu^{\prime}$ on one side of zero must continue rather globally. If the algebraic multiplicity $m$ is greater than or equal to three, we see that, if $\mu^{\prime}$ is non-zero and small, there must always be at least one eigenvalue with negative real part and there must be instability. If in addition, $m$ is odd, we easily see that the number of small negative real eigenvalues changes by 1 as $\mu^{\prime}$ crosses zero (as does the number of small eigenvalues with negative real part). If $m$ is even (including $m=2$ ) the number of small negative real eigenvalues still changes by 1 as $\mu^{\prime}$ crosses zero but the first order terms are insufficient to determine the number of small eigenvalues with negative real part for $\mu^{\prime}$ on one side of zero (because there is a pair of eigenvalues which are purely imaginary to first order). Note that this shows the delicacy of some of Wei's results. 
Note that our analysis for $m>2$ and Wei's stability result for $r=2$ shows that the algebraic multiplicity is 2 when $p=1+4 / N, r=2$ and $\gamma_{0}=1$. By continuity, it is still true if $r$ is close to 2 .

We need to justify our form for the bifurcation equation. Here, as in [4], it is convenient to choose a complement to the kernel of $L_{0}$ carefully respecting the Jordan chain structure of the eigenvalue zero of the linear operator $L_{0}$. If one does this and for $\mu^{\prime}=0$ uses the argument in $\S 8$ of Dancer [4], one finds that the bifurcation equation for $\mu^{\prime}=0$ is exactly $\delta^{m}$. Since our bifurcation equation will be smooth in $\delta$ and $\mu^{\prime}$, it then suffices to calculate the term linear in $\mu^{\prime}$ (and independent of $\delta$ ). To do this, we need to look at the construction of the bifurcation equation a little more closely. Let $P$ be a projection onto $R\left(L_{0}\right)$ and let $M$ be a complement to $N\left(L_{0}\right)=\operatorname{span}\{w\}$. Now for $\mu^{\prime}$ near zero and a small eigenvalue $\delta$ any eigenvector must be close to $N\left(L_{0}\right)$. Hence it suffices to look for solutions $w+m$ where $m \in M$ and $m$ is small. Now by the implicit function theorem, the equation $P L\left(\mu^{\prime}, \sigma\right)(m+w)=0$ can be solved for $m$ as a function of $\mu$ and $\delta, m=m\left(\mu^{\prime}, \delta\right)$, and $\|m\| \leq k\left(\left|\mu^{\prime}\right|+|\delta|\right)$. Here $L\left(\mu^{\prime}, \delta\right)=L_{\delta}$ for $\mu=\mu_{0}+\mu^{\prime}$ and $\tau=0$. Then the bifurcation equation is $\tilde{f}\left(L\left(\mu^{\prime}, \delta\right)\left(w+m\left(\mu^{\prime}, \delta\right)\right)=0\right.$ where $\tilde{f}$ spans $N\left(L_{0}^{*}\right)$. If we note $\tilde{f}(L(0,0))=0$, we see that terms linear in $\mu^{\prime}$ can only come from $\tilde{f}\left(\left(L\left(\mu^{\prime}, 0\right)-L(0,0)\right) w\right)$. Since $\tilde{f}$ is a multiple of $L_{0}^{-1}\left(w^{r-1}\right)$, we see that the term linear in $\mu^{\prime}$ is simply

$$
a \equiv f_{1}(w)\left(w^{p}, L_{0}^{-1} w^{r-1}\right)
$$

(by the formula for $L\left(\mu^{\prime}, \delta\right)$ ). It is easy to check that $f_{1}(w)>0$, since $w(R)>0$ for all $R \geq 0$. Now, by self-adjointness, $\left(w^{p}, L_{0}^{-1} w^{r-1}\right)=\left(L_{0}^{-1} w^{p}, w^{r-1}\right)=$ $-(p-1)^{-1} \int w^{r} \neq 0$. Here we have used $L_{0}^{-1}\left(w^{p}\right)=-(p-1)^{-1} w$ which we noted earlier. Hence $a \neq 0$ and we have proved our claim on the bifurcation equation.

We now use our ideas to obtain instability results for $\tau=0$ (and hence by continuity also for small positive $\tau$ ). Remember if we can find a region $T$ in parameter space where 0 is never an eigenvalue of $L_{\alpha}$ for any point of $T$, the sum of the multiplicities of the negative real eigenvalues is odd, then $L_{\alpha}$ has an eigenvalue with negative real part for every point of $T$. Now zero is only an eigenvalue if $\frac{q r}{(s+1)(p-1)}=1$ and hence we need to look at the two components of $Z=\{(q, r, s, p, \tau): q r \neq(s+1)(p-1)\}$. It is easily seen that there are only 2 components by looking at $q$ has a parameter. (By the same argument, there are only two components of $Z \cap\{\tau=0\})$. We know from Wei's work (or implicitly in [9]), that the component where $\gamma_{0}>1$ contains stable points and hence, by our remarks above the number of negative real eigenvalues is even if $\gamma_{0}>1$. By crossing $\gamma_{0}=1$ at a simple eigenvalue, it follows that the number of negative real eigenvalues is odd if $\gamma_{0}<1$ and there is always a negative real eigenvalue if $\gamma_{0}<1$ and $\tau \geq 0$ (and hence there is instability in this case).

We now make a more careful analysis of the signs of the coefficients of the crossing when $\mu=\mu_{0}$ and $\tau=0$ in the case of multiplicity 1 (and using our earlier bifurcation arguments) to obtain more cases of instability. When $\mu=\mu_{0}$, we can take the kernel to be spanned by $w$ and the adjoint to be spanned by $\left(L^{\prime}\right)^{-1}\left(w^{r-1}\right)$. Remember that $L^{\prime}$ is invertible on $L_{r}^{2}$. In this case it is easy to see from our earlier discussion that the bifurcation equation becomes 


$$
\mu^{\prime} f_{1}(w)\left(w^{p},\left(L^{\prime}\right)^{-1}\left(w^{r-1}\right)\right)-\delta\left(w,\left(L^{\prime}\right)^{-1}\left(w^{r-1}\right)+h o t=0\right.
$$

As we noted earlier,

$$
\begin{aligned}
f_{1}(w)\left(w^{p},\left(L^{\prime}\right)^{-1}\left(w^{r-1}\right)\right) & =-f_{1}(w)(p-1)^{-1}\left(w, w^{r-1}\right)<0 \\
\text { while }\left(w,\left(L^{\prime}\right)^{-1}\left(w^{r-1}\right)\right) & =\left(\left(L^{\prime}\right)^{-1}(w), w^{r-1}\right) \\
& =\left[(p-1)^{-1}-\frac{1}{2} N r^{-1}\right]\left(w, w^{r-1}\right)
\end{aligned}
$$

Hence we see that the direction the eigenvalue $\delta$ moves across zero as we increase $\mu^{\prime}$ across zero depends on the sign of $N(p-1)-2 r$ and the eigenvalue decreases (increases) across zero with increasing $\mu$ if $N(p-1)<2 r(N(p-1)>2 r)$. In particular we see that there is a small real negative eigenvalue (and hence there is instability) for $\mu$ slightly larger than $(p-1)$ if $p>1+2 N^{-1} r$. In particular if $r=2$, this shows we can have instability if $p>1+4 / N$ which shows a result of Wei's in [10] is in a sense best possible. Note that as we increase $\tau$ stability can only be regained by the negative real eigenvalues coalescing and then crossing the imaginary axis (necessarily not at zero). This is not really a local phenomenon. Indeed we suspect stability is never regained by increasing $\tau$. By a similar but more complicated calculation it can be shown that if $\tau>0$, we have instability for $\mu$ a little greater than $\mu_{0}$ if

$$
(s+1)^{-2} q r \tau-1+\frac{1}{2}(p-1) N r^{-1}>0
$$

and there may be stability if the inequality is reversed.

We now consider the case of large $\tau$. We show on $L_{r}^{2}$ that there is one or two negative eigenvalues and all the remainder of the spectrum lies strictly in the half plane $\operatorname{Re} \alpha>0$. Thus there is always instability. Moreover there is one negative real eigenvalue if $(p-1) \gamma_{0}<1$ and two if $(p-1) \gamma_{0}>1$. To prove these results, we will confine ourselves to the region $\operatorname{Re} \alpha \leq \frac{1}{2} \tau^{-1}$ which ensures that $\frac{q r}{s+1-\tau \alpha}$ stays bounded. Now the eigenvalues are given by the solutions of $\frac{q r}{s+1-\tau \alpha} f_{1}\left(\left(L^{\prime}-\alpha I\right)^{-1} w^{p}\right)=-1$. Now unless $\alpha$ is close to zero $\frac{q r}{s+1-\tau \alpha}$ is small for large $\tau$ while $\left(L^{\prime}-\alpha I\right)^{-1}$ is uniformly bounded on $\operatorname{Re} \alpha \leq \frac{1}{2} \tau^{-1}$ except near $\lambda_{1}$. (By standard resolvent estimates, $\left(L^{\prime}-\alpha I\right)^{-1}$ is uniformly bounded on our set except near $\lambda_{1}$ ). Hence we see that, for large $\tau$, eigenvalues can only occur near $\lambda_{1}$ or zero. Near $\alpha=\lambda_{1}, \frac{q r}{s+1-\tau \alpha}$ is an analytic function of $\tau^{-1}$ vanishing when $\tau^{-1}=0$. Moreover $\lambda_{1}$ is a simple eigenvalue of $L^{\prime} z=\alpha z$. Hence by standard perturbation theory (as in [6]), for large $\tau$ there is unique eigenvalue near $\lambda_{1}$ which is simple (and necessarily real).

To discuss eigenvalues near zero, we use (8). For $\alpha$ small, $f_{1}\left(\left(L^{\prime}-\alpha I\right)^{-1} w^{p}\right)$ is near $f_{1}\left(\left(L^{\prime}\right)^{-1} w^{p}\right)=-(p-1)^{-1}$ by earlier. Hence $\frac{q r}{s+1-\tau \alpha}$ is close to $(p-1)^{-1}$ and hence $\tau \alpha$ is close to $\tilde{\mu}_{0}$ where 


$$
\frac{q r}{s+1-\widetilde{\mu}_{0}}=(p-1)^{-1} .
$$

If we then use $\ell=\tau \alpha$ as the new variable (rather than $\alpha$ ), we have the equation $\left.\frac{q r}{s+1-\ell} f_{1}\left(L^{\prime}-\ell \tau^{-1}\right)^{-1} w^{p}\right)=-1$. We use the implicit function theorem (on $\mathbb{C}$ ) to show that this has a unique solution $\ell$ near $\widetilde{\mu}_{0}$ for $\tau$ large. This proves our claim. We see that this solution is positive if $\frac{q r}{s+1}<(p-1)^{-1}$ and is negative if $\frac{q r}{s+1}>(p-1)^{-1}$. This proves our claim.

Next we see that it is a very frequent occurrence that as we vary parameters, we obtain a curve on which non-real eigenvalues of (4) cross the imaginary axis. We choose an example where (4) for $\tau=0$ has no eigenvalues with non positive real part and $\gamma_{0}>1$ and we then increase $\tau$. For large $\tau$ there are exactly two real negative eigenvalues. Thus by continuity of eigenvalues, as we vary $\tau$, eigenvalues must cross the imaginary axis at non-zero points. By our earlier results this must occur with $\tau$ neither small not large. We can use an analyticity argument as at the bottom of p25 of [3] to deduce that when the crossing occurs, there is strict crossing of the imaginary axis. (In particular, there are only eigenvalues in $L_{r}^{2}$ on the imaginary axis for isolated values of $\tau$ ). We use this in Section 2 to obtain Hopf type bifurcations. Finally note that these examples imply that in some cases the eigenvalue of (4) of smallest real part are complex.

Note that there is a completely analogous theory for (4) on a half space (which is equivalent to (4) on $R^{N}$ where our space of functions are even in one variable). The problem still reduces as before and indeed the spectrum with $\alpha$ real and $\alpha<0$ or $\operatorname{Im} \alpha \neq 0$ and $\tau \operatorname{Re} \alpha<1+s$ is the same as before (as is its multiplicity).

Finally, we want to see how some of these ideas can be used to simplify Wei's calculations. If $\tau=0$ and $r=p+1$, our operator is self-adjoint (for example, it is easy to prove $\left(L_{0} u, \bar{u}\right)$ is always real) and hence the eigenvalues are real. Thus, by our earlier remarks it suffices to look for real eigenvalues of $(3)$ in $\left(\lambda_{1}, 0\right)$ with radial eigenfunctions. We then need to follow the argument in the four lines following (5.17) in $[10]$.

In the case where $r=2$ and $\tau=0$, Wei's argument can be simplified a little because we need only work in the space of radial functions and the kernels are much smaller which simplifies the arguments.

2. Results for the original equation and Hopf bifurcation. The purpose of this short section is to prove that eigenvalues of the limit equation (4) generate nearly eigenvalues of (3) and conversely. We also consider Hopf bifurcations. We mainly consider the interior peak case.

It is convenient to consider rather than (3) the rescaled equation

$$
\begin{gathered}
-\Delta h+h-p \widetilde{u}_{\epsilon}^{p-1} h+f_{1, \epsilon}(h) \widetilde{u}_{\epsilon}^{p}=\alpha h \\
\text { on } \widetilde{\Omega}_{\epsilon} \\
h=0 \text { on } \partial \widetilde{\Omega}_{\epsilon}
\end{gathered}
$$

where $\widetilde{\Omega}_{\epsilon}=\left\{\epsilon^{-1}\left(x-x_{\epsilon}\right): x \in \Omega\right\}$, and where 
$f_{1, \epsilon}(h)=\frac{q r}{s+1-\tau \alpha}\left(\int_{\widetilde{\Omega}_{\epsilon}} \widetilde{u}_{\epsilon}^{r}\right)^{-1} \int_{\widetilde{\Omega}_{\epsilon}} \tilde{u}_{\epsilon}^{r-1} h, x_{\epsilon}$ is the peak point of $u_{\epsilon}, \widetilde{u}_{\epsilon}=u_{\epsilon}(\epsilon(x-$ $\left.x_{\epsilon}\right)$ ) and we are working on the space $L^{2}\left(\widetilde{\Omega}_{\epsilon}\right)$. It is convenient to write this equation in the form

$$
h=R_{\epsilon}(\alpha)\left(-p \widetilde{u}_{\epsilon}^{p-1} h+f_{1, \epsilon}(h) \widetilde{u}_{\epsilon}^{p}\right) \equiv Z_{\epsilon}(\alpha)(h)
$$

where $R_{\epsilon}(\alpha)$ is the inverse of $-\Delta+(1-\alpha) I$ with Neumann boundary conditions on $\widetilde{\Omega}_{\epsilon}$ (which exists if $\operatorname{Re} \alpha<1$ or $\operatorname{Im} \alpha \neq 0$ ). We then consider the mapping $\widetilde{Z}_{\epsilon}(\alpha)=$ $i_{\epsilon} Z_{\epsilon}(\alpha) P_{\epsilon}$ on $L^{2}\left(R^{N}\right)$ where $P_{\epsilon}$ is the natural projection of $L^{2}\left(R^{n}\right)$ on $L^{2}\left(\widetilde{\Omega}_{\epsilon}\right)$ (by restriction) and $L_{\epsilon}$ is the natural inclusion of $L^{2}\left(\Omega_{\epsilon}\right)$ into $L^{2}\left(R^{N}\right)$. (In fact $L_{\epsilon}=P_{\epsilon}^{*}$ ). Since $\widetilde{u}_{\epsilon}$ converges to $w$ in $L^{2}\left(R^{N}\right)$ (and $L^{\infty}\left(R^{N}\right)$ as follows easily from the estimates in [11] and [7]), we see easily that $\widetilde{Z}_{\epsilon}(\alpha) h \longrightarrow Z(\alpha) h$ for each $h$ in $L^{2}\left(R^{N}\right)$, if we prove that $\left\|R_{\epsilon}\right\|$ are uniformly bounded (for $R e \alpha \leq 1-\delta$ ) and $R_{\epsilon}(\alpha) h \longrightarrow R(\alpha) h$ in $L^{2}\left(R^{N}\right)$ for every $h \in C_{0}^{\infty}\left(R^{n}\right)$. Here $Z=R(\alpha)\left(-p w^{p-1} h+\gamma(\alpha) f(h) w\right)$, where $R$ is the inverse of $-\Delta+(1-\alpha) I$ on $L^{2}\left(R^{n}\right)$. Remember that $A_{\epsilon} h \longrightarrow A h$ as $\epsilon \longrightarrow 0$ for every $h \in L^{2}\left(R^{n}\right)$ if $\left\|A_{\epsilon}\right\|$ are uniformly bounded and the convergence holds for $h$ in a dense set. (Note that the resolvent equation for $R(\alpha)$ then ensures that the map $\alpha \longrightarrow R(\alpha)$ is uniformly continuous and thus the $\alpha$ variation causes no difficulties). The uniform estimate for $\left\|R_{\epsilon}(\alpha)\right\|$ holds from trivial estimates (and in fact $R_{\epsilon}(\alpha)$ are uniformly bounded as maps from $L^{2}\left(\Omega_{\epsilon}\right)$ to $W^{1,2}\left(\Omega_{\epsilon}\right)$ ). We simply multiply the equation for $R_{\epsilon}(h)$ by $R_{\epsilon}(h)$ and take the real part. By using the weak form of the equation for $R_{\epsilon}(\alpha) h$, it is easy to check that $R_{\epsilon}(\alpha) h$ converges weakly to $R(\alpha) h$ in $L^{2}\left(R^{n}\right)$ as $\epsilon$ tends to zero and hence, by the Sobolev embedding theorem there is strong convergence in $L^{2}$ on compact sets in $R^{n}$. Hence we see that it suffices to prove that $\int_{\|x\| \geq K}\left\|R_{\epsilon}(\alpha) h\right\|^{2} \longrightarrow 0$ as $K \longrightarrow \infty$ uniformly in $\epsilon$. Choose $\ell$ smooth such that $\ell=1$ if $\|x\| \geq K$ and $\ell h=0$. Recall that $h$ has compact support. We scalar multiply the equation for $v_{\epsilon}=R_{\epsilon}(\alpha) h$ by $\ell^{2} \bar{v}_{\epsilon}$ and take the real part. After a simple calculation, we find that inf $\{1, \operatorname{Re}(1-\alpha)\}\left\|\ell v_{\epsilon}\right\|_{1,2}^{2} \leq \int_{\widetilde{\Omega}_{\epsilon}}\left|v_{\epsilon}\right|^{2}|\nabla \ell|^{2} \leq K_{1} \int_{\widetilde{\Omega}_{\epsilon} \cap B_{k}}\left|v_{\epsilon}\right|^{2} \leq K_{1}\left\|v_{\epsilon}\right\|_{2}^{2}$ where $K_{1}^{\frac{1}{2}}=\sup |\nabla \ell|$. Since we can choose $K_{1}$ to be small if $K$ is large, we see that if $K$ is large $\left\|\ell v_{\epsilon}\right\|_{2}$ is small and our claim follows.

We now prove that $\widetilde{Z}_{\epsilon}(\alpha)$ are a collective compact set of operators in the sense of [1] for $\alpha$ in a suitable set in $\mathbb{C}$. We first prove that, if $T$ is bounded in $L^{2}(\Omega)$ and $W$ is a compact set in $\mathbb{C}$, then

$$
\left\{R_{\epsilon}(\alpha)\left(-p \widetilde{u}_{\epsilon}^{p-1} h+f_{1, \epsilon}(h) \widetilde{u}_{\epsilon}^{p}\right): 0<\epsilon \leq \epsilon_{0}, \alpha \in W, x \in \mathbb{C}\right\}
$$

lies in a compact subset of $L^{2}\left(R^{n}\right)$. Our expression splits naturally into two terms. It suffices to separately prove the compactness for each term. We first consider the second term which is the easier. By the properties of $R_{\epsilon}(\alpha)$ proved a little earlier, it suffices to prove that $\left\{f_{1, \epsilon}(h) \widetilde{u}_{\epsilon}^{p}\right\}$ lies in a compact set. Since $\widetilde{u}_{\epsilon}$ (or more strictly $i_{\epsilon} \widetilde{u}_{\epsilon}$ ) converges to $w$ in $L^{2}\left(R^{n}\right)$ and since $\left\|f_{1, \epsilon}\right\|$ are uniformly bounded, the result follows easily.

We now return to the first term. First note that if, $m$ has compact support in $R^{n}$, then the map $h \longrightarrow R(\alpha)(m h)$ is compact on $L^{2}\left(R^{N}\right)$. This follows by very 
similar arguments to those used in our proof that $R_{\epsilon}(\alpha) h \longrightarrow R(\alpha) h$ as $\epsilon \longrightarrow 0$ if $h \in C_{0}^{\infty}\left(R^{N}\right)$. (In particular, we use the test function argument to prove the uniform (in $h$ ) decay at infinity). Since we can write $m \in C_{0}\left(R^{N}\right)$ (that is the continuous functions in $R^{n}$ tending uniformly to zero at infinity) as $m=m_{1}+m_{2}$ where $m_{1}$ has compact support and $\left|m_{2}\right| \leq \tilde{\epsilon}$ on $R^{N}$, it follows easily that the map $h \longrightarrow$ $R(\alpha)(m h)$ is compact on $R^{n}$ if $m \in C_{0}\left(R^{N}\right)$. Essentially the same argument shows that $\left\{R_{\epsilon}(\alpha)(m h): h \in L^{2}\left(R^{N}\right),\|h\|_{2} \leq 1,0<\epsilon \leq \epsilon_{0}\right\}$ is compact if $m \in C_{0}\left(R^{N}\right)$. With these results, it is now easy to check the compactness of the first term. Hence the collective compactness follows.

We can now apply Lemma 4 in [4] to prove that near each eigenvalue of (4) with $\operatorname{Re} \alpha<1$, there is least on eigenvalue of (3) for all small $\epsilon$ (and in fact the local sum of multiplicities of eigenvalues is preserved). (It is possible to prove a similar result holds if $\operatorname{Im} \alpha \neq 0$ with a similar proof). As noted in [10], the converse follows easily by normalizing eigenfunctions $h$ in $L^{2}\left(\Omega_{\epsilon}\right)$ to have $\|h\|_{\infty}$ equal to 1 and then using a blow up argument where $|h|$ has its maximum.

Finally using again the estimate that if $L$ is self-adjoint,
$\left\|(L-B I)^{-1}\right\| \leq \frac{1}{\operatorname{distance}(B, \sigma(L))}$ it is easy to see our bounds in Section 1 for the spectrum of (3) in Re $\alpha \leq 1$ hold uniformly in $\epsilon$. (We use the same arguments as we did to bound the spectrum of (4)). Hence we have established the following theorem.

THEOREM 1. (i) If $\alpha$ is an eigenvalue of (4) on $L_{r}^{2}$ with $R e \alpha<1$, for all small $\epsilon$, there is an eigenvalue $\alpha_{\epsilon}$ of (3) near $\alpha$. Conversely, if $\alpha_{\epsilon(n)}$ are eigenvalues of (3) with $\operatorname{Re} \alpha_{\epsilon(n)} \leq B<1$ for all $n$, then a subsequence of $\alpha_{\epsilon(n)}$ converge to an eigenvalue of (4) on $L_{r}^{2}$.

(ii) If $B<1$ and (4) on $L^{2}$ has no eigenvalue with $\operatorname{Re} \lambda=B$ then for small $\epsilon$, the number of eigenvalues with Re $\lambda<B$ counting multiplicity is the same for (4) and (3).

REMARKS. It is easy to see that the results holds locally uniformly in the parameters. We could prove results for curves of eigenvalues but we do not meet it. (The formulation needs care because eigenvalues may split). There is an analogous result for boundary peak solutions where we replace (4) by a half space problem with a Neumann boundary condition (or equivalently to a problem on $R^{n}$ but where we restrict our functions to even in one variable). Note that this only affects the spectrum by changing the multiplicity of zero. There are similar results for Dirichlet problems with an interior peak (where the proofs can be simplified a bit) or for multipeak solutions. The last result needs considerably more care. For example, in the case of 2 boundary peaks not close and peaks both near non-degenerate critical points of the mean curvature on $\partial \Omega$, the non-small eigenvalues of (3) with $\operatorname{Re} \alpha \leq \delta$ (where $\delta$ is small and positive) are close to those of (4) with the same multiplicity or to $\lambda_{1}$ (and thus the solution is unstable). The methods can also be used to study more than 2 peaks and cases where some peaks are boundary peaks and some interior peaks.

We now obtain Hopf bifurcation. We consider the analytic curve $y(t)$ we constructed at the end of Section 2 so that (4) on $L_{r}^{2}$ has no eigenvalues with non-positive real part for $t=0$, exactly 2 (both real) for $t=1$ and no zero eigenvalue for all $t \in[0,1]$. By our last result, if $\epsilon$ is small, (3) along the curve $y(t)$ will have 2 negative real eigenvalues which are not small for $t=0$, no small eigenvalues with non-positive 
real part for $t=1$ and exactly $n$ small eigenvalues for counting multiplicity all $t$ which stay separated from the rest of the spectrum. We now argue as in Section 1 with the eigenvalues which are not small to show that along $y(t)$ for fixed small positive $\epsilon$ there are only non-small purely imaginary eigenvalues for isolated values of $t$ and there is a strict net crossing of the imaginary axis of non-small eigenvalues as we vary $t$ from 0 to 1 . We can then apply global Hopf bifurcations theorems to the non small purely imaginary eigenvalues of (3) provided we know that 0 is not an eigenvalue of (3). As we will see in a moment, this holds in many cases. Assuming this condition holds, we can apply global Hopf bifurcation theorems exactly as on p26 of [3] to obtain a global branch of positive periodic solutions. They continue to large solutions or collapse on to another stationary solution or the period of the solutions on the branch becomes large. Here we are using $\tau$ as a parameter. Note that when we apply the Hopf bifurcation techniques we only need to look at purely imaginary eigenvalues which are a positive integer multiple of the given one so that small purely imaginary eigenvalues do not affect the argument. These periodic solutions, when they bifurcate, bifurcate for $\tau$ close to a point where (2) has purely imaginary eigenvalues and the period of the solutions will be neither small nor large (because it is determined by the imaginary part of the non small purely imaginary eigenvalues. Thus our bifurcating purely periodic solutions will be peaked in the space variables (with peak close to that of the stationary solution) and the time period will be neither small nor large and is determined by (4) at least asymptotically. Note that we could use more general analytic curves (where the other parameters depend on $\tau$ ). This is much more general than the results in [9] because we prove bifurcation to periodic solutions much more generally, our bifurcation is much more global and we keep better control of the solutions. We suspect that these solutions are sometimes stable in the boundary peak case and meta stable in the inteior peak case. (At a classical Hopf bifurcation point, this is determined by the direction of bifurcation).

It remains to check when the invertibility conditions holds. For a boundary peak solution, this holds if the solutions peak close to a non-degenerate critical point of the mean curvature on $\partial \Omega$ and in the interior peak case if the peak is close to a nondegenerate peak point in the sense of [10] (cp [10] and [11]). Note that it is much easier to prove that zero is not an eigenvalue than to determine the small eigenvalues. Note also that Wei's results in [10] and [11] (when combined with the ideas in the appendix to [10]) imply that in fact there are no small purely imaginary eigenvalues when there is non-degeneracy so our bifurcating branch can not return to where it bifurcates with solutions of large minimal period.

Note that when these conditions fails, but $\Omega$ is symmetric one can sometimes regain invertibility by working in a symmetric subspace (as in [2]). For example, this method can be used for the domains in [2]. Note also that we could prove Hopf bifurcation along much more general curves in parameter space.

In the two peak case mentioned after Theorem 1, it is possible to modify our argument to prove that there is also Hopf bifurcation (at parameters close to the ones for which one peak Hopf bifurcation occurs) and the solutions here have the two peaks oscillating almost in phase. This can also be done for more than 2 peaks.

Our methods are quite different to those of [9] where they instead reduce to a complicated scalar equation depending on $\epsilon$ (which is similar to (8)). We obtain a limiting problem independent of $\epsilon$ (which already gives information on the limit of 
quantities in [9] as $\epsilon$ tends to zero), our methods seem more flexible than those in [9] and show that asymptotically the boundary and interior peak solution case have the same non-small complex eigenvalues. Moreover, we obtain much better information on the solutions when there are Hopf bifurcations and we prove Hopf bifurcations much more generally (and more globally). Our results are complementary to those in [10].

\section{REFERENCES}

[1] N. Anselone, Collectively compact operator approximation theory and applications to integral equations, Prentice Hall, Englewood Cliffs, 1971.

[2] E.N. DANCER, On the uniqueness of the positive solution of a singularly perturbed problem, Rocky Mountain J. Math, 25 (1995), pp. 957-975.

[3] E.N. DANCER, On uniqueness and stability for solutions of singularly perturbed predator-prey type equations with diffusion, J. Diff. Eqns., 102 (1993), pp. 1-32.

[4] E.N. DANCER, Bifurcation theory in real Banach space, Proc London Math Soc., 23 (1971), pp. 699-734.

[5] J. IZE, Bifurcation theory for Fredholm operators, Memoirs Amer Math Soc., 174 (1976).

[6] I. GoKHBERg AND M. KREIN, Fundamental theorems on deficiency indices, root numbers and indices of linear operators, Amer Math Soc. Transls, (2) 13, 1960, pp. 185-264.

[7] T. KAYO, Perturbation theory for linear operators second edition, Springer Verlag, Berlin, 1976.

[8] W.M. NI AND I. TAKAGI, Locating the peaks of least energy solutions to a semilinear Neumann problem, Duke Math J., 70 (1993), pp. 247-281.

[9] W.M. Ni, I. TAKAgi AND E. YANAGIDA, Stability analysis of point condensation solutions to a reaction diffusion system proposed by Gierer and Meinhardt, preprint.

[10] J. WEI, On single interior spike solutions of Gierer-Meinhardt systems: uniqueness and spectral estimates, European J. App. Math, 10 (1999), pp. 353-378.

[11] J. WEI, Uniqueness and eigenvalue estimates of boundary spikes solutions, preprint. 